\title{
Cracking behaviour of fine-grained soils: from laboratory testing to numerical modelling
}

\author{
Pierre Gerard ${ }^{1, *}$, Ian Murray ${ }^{2}$, and Alessandro Tarantino ${ }^{2}$ \\ ${ }^{1}$ Université Libre de Bruxelles (ULB), BATir Department, Brussels, Belgium \\ ${ }^{2}$ University of Strathclyde, Department of Civil \& Environmental Engineering, Glasgow, UK
}

\begin{abstract}
Many experimental evidences suggest that desiccation cracks in clay initiate as a result of the mobilization of soil tensile strength. However this mechanical approach disregards the cohesionless and effective stress-dependent behaviour of fine-grained soil. On the other hand recent findings in the literature suggest that effective stress-dependent shear failure criteria would be appropriate to explain the mechanisms of desiccation cracking for tensile total stress states. This work aims at assessing the validity of a shear failure criterion to predict the onset of cracking in clay forms exposed to air drying. Clay forms of various geometries were experimentally subjected to non-uniform hydraulic and mechanical boundary conditions. Time and location for crack initiation are monitored using a digital camera. Cracking experiments are then modelled in a hydro-mechanical framework using an effective-stress shear failure criterion. The comparison of simulations with experimental results for both the time and the location of cracking allows assuming that cracking occurs due to failure in shearing.
\end{abstract}

\section{Introduction}

Tension cracks in fine-grained soils owing to desiccationinduced shrinkage is a key issue in many geotechnical applications, as stability of natural slopes [1] or flood embankments [2]. Understanding the mechanisms of failure in tension in clay soils is therefore necessary to predict tension crack initiation and propagation. At the scale of the representative elementary volume, desiccation cracking has been extensively investigated experimentally $[3,4]$. Nonetheless, the mechanisms of crack initiation are not yet well understood. Experimental evidence relating to desiccation cracking indicates that crack initiation occurs in Mode I (i.e. opening mode), suggesting that crack initiation occurs when total tensile stresses exceed the soil tensile strength [3;5-7].

However, this mechanical approach disregards the effective stress dependent behaviour of soils generally considered in modern soils mechanics. To be able to analyse the onset of tension cracks in terms of effective stress states Thusyanthan et al. [8] performed four points bending on saturated clay bars equipped with high capacity tensiometers measuring negative pore water pressures. The combination of the effective stress states in the extreme tension fibre of the different bars at the initiation of failure allows defining a shear failure envelope similar to the one observed under triaxial compression. More recently Murray et al. [9] extended those conclusions to unsaturated specimen. They performed direct tensile tests on a series of initially saturated and unsaturated clay samples prepared at different initial suctions. The use of high-capacity tensiometers allowed also interpreting failure conditions in terms of the effective stress. The results suggest that mechanism of failure under tensile total stress states can be interpreted in terms of a cohesionless and effectivesstress dependent shear failure criterion, both for initially saturated and unsaturated specimen (Mode II).

This paper presents further experimental investigations to assess the relevance of an effective stress dependent shear failure criterion to explain the mechanisms and predict the onset of cracking under tensile total stress states. It is proposed to carry out desiccation tests on a series of bars with complex geometries and non-uniform hydraulic and mechanical boundaries conditions. Time and location for crack initiation are monitored using a digital camera. Cracking experiments are then tentatively modelled in a hydromechanical framework using an effective stress shear failure criterion. The comparison of simulations with experimental results for both the time and the location of cracking allows assuming that cracking occurs due to failure in shearing.

\section{Material and sample preparation}

The material used for the experimental programme is a Vitreous China (VC) mix, commonly used in the ceramic industry. The silt and clay fractions are respectively 0.70 and 0.30 .

The clay was reconstituted to slurry state and specimens were created by casting the slurry (slip) into the plaster mould. After consolidation, the specimen of dimensions shown in Figure 1 was removed from the

\footnotetext{
*Corresponding author: piergera@ulb.ac.be
} 
mould. The specimen was then vacuum sealed and stored for 24 hours. The specimen was then removed and then weighed, allowing for a calculation of the weight of solid material in the specimen. Slip casting was chosen as the method of creating specimens as it was the simplest method of creating identical test specimens of the relatively complex geometry required for the tests.

\section{Evaporation tests}

\subsection{Experimental set-up}

Desiccation tests involved specimens with complex geometry and non-uniform hydraulic and mechanical boundary conditions. First shrinkage is induced through the air-drying of the specimen. Then the specificity of the tests was to partially prevent the shrinkage-induced deformations to generate total tensile stress within the samples. The shrinkage is actually prevented by the specimen itself, using a "double T" specimen geometry and using the end sections to provide the resistance to axial shrinkage. The test apparatus has therefore 4 faces of restraint (made of Teflon to allow free lateral shrinkage), acting to prevent axial shrinkage at each arm of the double $\mathrm{T}$ specimen. The specimen sits on a layer of ball bearings which negate any frictional effect between the specimen and the base. The equipment is placed on a balance which measures mass loss throughout the test. A digital camera is used in con-junction with time lapse imaging software to record the location and time of cracking.

The geometry of the specimen is given in Figure 1 (height of the sample is $10 \mathrm{~mm}$ ).

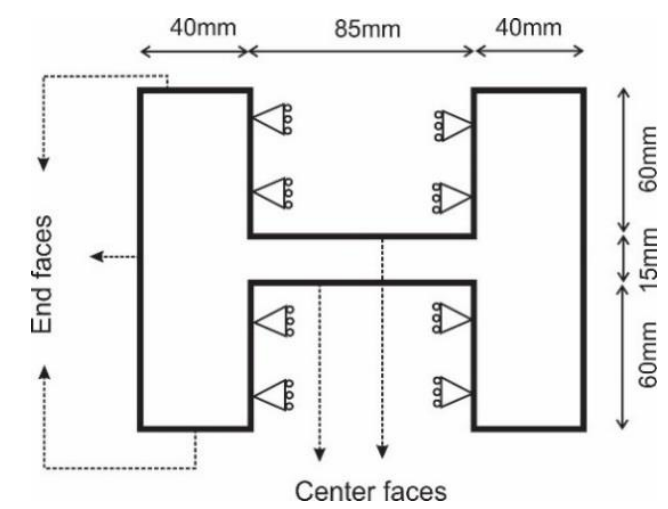

Fig. 1. Geometry of the specimen.

\subsection{Results}

Four desiccation tests were performed with various drying conditions (presence or not of greased faces, different relative humidity of the air $\mathrm{RH}$, different initial water content of the bar). Identical mechanical restraints are imposed in all the tests. The top surface is always greased. The experimental conditions are summarized in Table 1.

The cracking is always observed first on the top surface, at the corner between the 'flange' and the 'web' (Figure 2). The times of cracking determined visually from the digital camera are given in Table 1. The cracking time from Test 4 cannot be clearly identified from the digital camera. However, the monitoring of the force at the restraints has recorded a decrease of the force after $2 \mathrm{~h} 18$ that can be reasonably considered as the indication of the initiation of cracking.

Table 1. Experimental conditions and time of cracking.

\begin{tabular}{c|c|c|c|c|c}
\hline $\begin{array}{c}\text { Test } \\
\text { No }\end{array}$ & $\begin{array}{c}\text { Initial } \\
\text { water } \\
\text { content }\end{array}$ & $\begin{array}{c}\text { Non- } \\
\text { greased } \\
\text { faces }\end{array}$ & $\begin{array}{c}\text { Greased } \\
\text { faces }\end{array}$ & RH & $\begin{array}{c}\text { Cracking } \\
\text { time }\end{array}$ \\
\hline 1 & $21.3 \%$ & End+Center & Top & $42 \%$ & $3 \mathrm{~h} 28$, \\
\hline 2 & $19.7 \%$ & End+Center & Top & $53 \%$ & $4 \mathrm{~h} 37$ \\
\hline 3 & $21.0 \%$ & End & Top+Center & $50 \%$ & $5 \mathrm{~h} 00$ \\
\hline 4 & $20.0 \%$ & Center & Top+End & $42 \%$ & $2 \mathrm{~h} 18$ \\
\hline
\end{tabular}

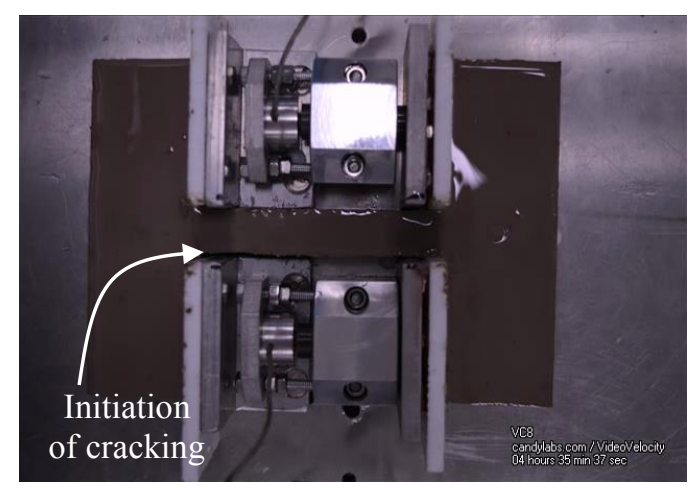

Fig. 2. Location of cracking.

\section{Numerical simulation}

\subsection{Geometry}

Complementary investigation has shown that evaporation from greased faces is reduced but not totally prevented (ratio between evaporation rates of greased and nongreased faces is around 8). 3D hydro-mechanical simulations are therefore required to properly reproduce the gradients of pore-water pressures that developed within the bars and the shrinkage thereof. Finite element code Lagamine is used $[10,11]$ to compare time and location of cracking simulated numerically with the ones observed experimentally (Table 1). The geometry of the model corresponds to the one defined in Figure 1, with a height of the sample equal to $10 \mathrm{~mm}$. Due to symmetry, only a quarter of the bar is modelled.

\subsection{Boundary conditions}

Vertical displacement is prevented at the bottom of the bar, and horizontal displacement is prevented perpendicularly to the restraints. Evaporation rate is prevented from the bottom of the sample and from the faces where restraints are installed. The evaporation rate is modelled through a convective evaporation condition:

$$
q=\alpha\left(\rho_{v, \Gamma}-\rho_{v, \text { air }}\right)
$$


where $\alpha$ is the mass transfer coefficient that is mainly controlled by the air circulation in proximity of the evaporation surface and can be assumed to remain constant during the desiccation test under controlled laboratory conditions $[11,12], \rho_{v, \Gamma}$ and $\rho_{v, \text { air }}$ are the vapour densities at the bar face and of the surrounding air, respectively. The vapour density is derived from the relative humidity via the ideal gas law. The mass transfer coefficient is fitted on the mass loss of the bar during the initial stage of evaporation where the vapour density $\rho_{v, \Gamma}$ is practically equal to the density of saturated vapour.

As explained above, a ratio around 8 between greased and non-greased faces is imposed for the mass transfer coefficient. Both mass transfer coefficients are kept the same for all the tests.

\subsection{Constitutive equations and parameters}

The fluid transfers within the clay are reproduced by the advection of the liquid phase (generalised Darcy's law) and the diffusion of the water vapour (Fick's law) [13]. The soil water retention curve is given by the van Genuchten's expression type [14]:

$$
S_{r e}=\frac{S_{r}-S_{r e s}}{1-S_{r e s}}=\left[1+\left(\frac{S}{P_{r}}\right)^{n}\right]^{\frac{1}{n}-1}
$$

where $S_{r}$ is the degree of saturation, $S_{r e s}$ is the residual degree of saturation, $S_{r e}$ is the effective degree of saturation, $s$ is the suction, $P_{r}$ and $\mathrm{n}$ are soil parameters. The soil parameters have been fitted on the water retention data from the Vitreous China mix [9], and the fitting provided $P_{r}=1430 \mathrm{kPa} ; n=2.4$ and $S_{\text {res }}=0.08$.

The unsaturated hydraulic conductivity $K$ is based on a modified Kozeny-Karman relationship. The saturated hydraulic conductivity is assumed to depend on void ratio $e$ (square bracket in Eq. 3) and a hyperbolic relationship of the degree of saturation $S_{r}$ is considered to model the decrease in hydraulic conductivity in the unsaturated domain:

$$
K=\left[K_{\text {sat }, \text { ref }}\left(\frac{e}{e_{\text {ref }}}\right)^{3}\left(\frac{1+e_{r e f}}{1+e}\right)\right]\left(S_{r}\right)^{\beta}
$$

From the constant head hydraulic conductivity test, a reference saturated hydraulic conductivity $K_{\text {sat,ref }}$ of 1.94 $10^{-11} \mathrm{~m} / \mathrm{s}$ was obtained at the reference void ratio $e_{r e f}=0.47$. The inverse analysis of the mass loss recorded during an evaporation test performed on an initially saturated cylindrical sample (with evaporation only from the top) returned a value for the material parameter $\beta=3.7$.

The mechanical constitutive law is written in terms of generalized effective stress $\sigma^{\prime}[15]$ :

$$
\sigma^{\prime}=\sigma+S_{r}^{\varsigma} s
$$

with $\sigma$ the total stress. In this expression, it has been decided to use a hyperbolic function of the degree of saturation for the expression of the effective stress parameter, as suggested by [16]. $\zeta$ is a material parameter.
Non-linear elasticity is considered for the mechanical behaviour of the Vitreous-China mix, assuming that the bars are initially normally consolidated:

$$
\begin{gathered}
d \varepsilon_{v}^{e}=\frac{\lambda}{1+e_{0}} \frac{d p^{\prime}}{p^{\prime}} \\
d \varepsilon_{d}^{e}=\frac{2(1+v)}{9 \lambda(1-2 v)} d q
\end{gathered}
$$

These are associated with a cohesionless shear failure criterion:

$$
\begin{gathered}
f \equiv q-M p^{\prime}=0 \\
M=6 \sin \varphi^{\prime} /\left(3-\sin \varphi^{\prime}\right)
\end{gathered}
$$

where $\varepsilon_{v}^{e}$ and $\varepsilon_{v}^{e}$ are the volumetric and deviatoric elastic strain respectively, $q$ the deviatoric stress, $p$ ' the mean effective stress, $\varphi$ ' the friction angle, $e_{0}$ the initial void ratio, $\lambda$ the slope of the normal consolidation line in the $\ln$ $p$ '-e plane and $v$ is the Poisson ratio (assumed equal to $0.3)$.

The slope of the normal consolidation line was derived from suction and void ratio measured in the saturated domain $(\lambda=0.058)$.

The failure criterion was derived from direct tensile tests on samples with different initial suctions where suction was monitored using high-capacity tensiometers [9]. The failure data could be satisfactorily reproduced by a failure envelope defined by a friction angle $\varphi^{\prime}=23,7^{\circ}$ and an effective stress parameter $\zeta=2$ (Fig. 4). It is here worth mentioning that the incorporation of a soil water retention curve depending also on the variation of the void ratio would probably allow a better reproduction of the failure data by the failure envelope on Fig.4.

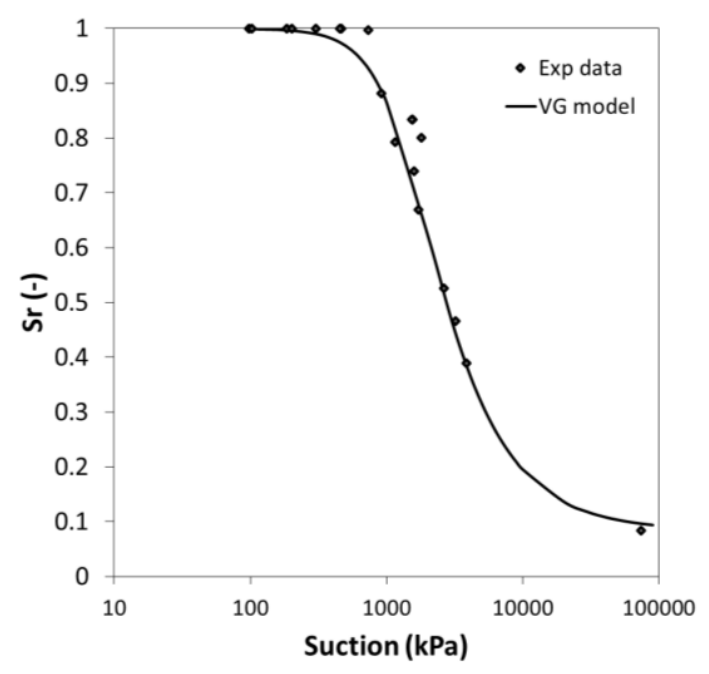

Fig. 3. Calibration of van Genuchten water retention curve model on retention data on Vitreous China mix and obtained by [9]. 


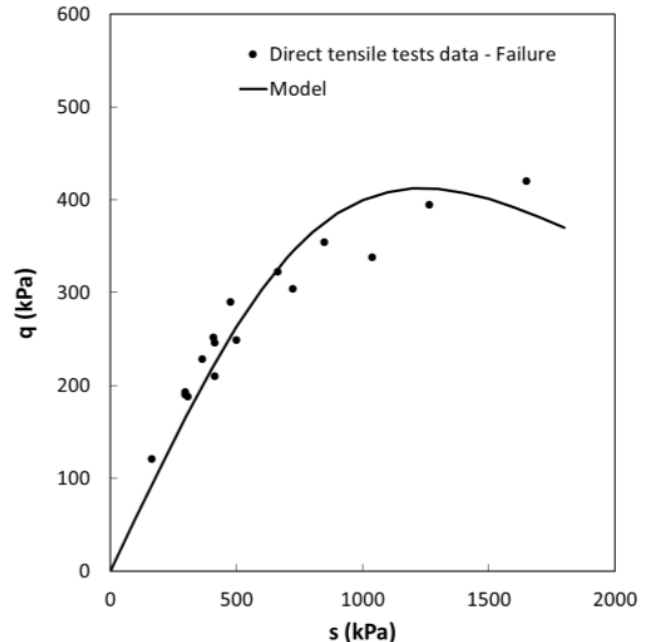

Fig. 4. Definition of effective-stress dependent failure criterion based on direct tensile tests data from [9].

\subsection{Initial conditions within the bar}

All the bars are initially normally consolidated. The initial suction $s_{0}$ within each bar is deduced from the initial water content (Table 1) and the soil water retention curve (Equation 2). The initial void ratio $e_{0}$ of each bar is then calculated from the normally consolidated line determined from the soil water retention data in the saturated domain:

$$
e_{0}=e^{*}-\lambda \cdot \ln p^{\prime}
$$

with $e^{*}=0.819$ and $\lambda=0.058$. Equation (3) allows determining the initial hydraulic conductivity of the material.

\subsection{Determination of mass transfer coefficients}

Mass transfer coefficients of non-greased and greased faces were calibrated from the mass loss of the bar during the initial stage of air-drying and assuming a ratio of 8.5 between the two coefficients. This ratio has been determined under controlled laboratory conditions by comparing 1-D evaporation rates from cylindrical samples with evaporation prevented on all the faces except the top one that was respectively greased and nongreased. It also appeared that evaporation rate from the centre faces of the bar (Figure 1) was impacted by the presence of measuring devices (ball load cells) in that zone which reduced the convection around the centre faces (see Figure 2). A good agreement between the mass loss monitored on the four air-dried bars and the results of simulated was obtained with a mass transfer coefficient $\alpha$ equal to $8.510^{-3} \mathrm{~m} / \mathrm{s}$ for the non-greased faces and $10^{-3}$ $\mathrm{m} / \mathrm{s}$ for the greased faces.

\subsection{Determination of time and location of cracking}

Hydro-mechanical modelling of the air-drying of the bars and the associated shrinkage is performed with the constitutive equations, set of soil parameters and drying conditions determined above. It is worth noticing that all soil parameters were determined with independent tests (see section 4.3). As a result, simulations can be considered as "blind predictions".

Analysis of the hydro-mechanical modelling of the desiccation provides the effective stress states within the bar throughout air-drying. It highlights that in the 4 test conditions the effective stress state reaches the shear failure envelope always first at the top surface of the bar, at the corner between the 'web' and the 'flange' (Figure 5). Such predictions are consistent with the experimental observations.

For the 4 tests, the ratio $\mathrm{q} / \mathrm{p}$ ' at the corner between the web and the flange is plotted versus the time and compared to the value of $M$ parameter defined by Equation 8. Figure 6 allows defining the time of cracking predicted by the model which is summarized and compared with experimental data in Table 2.

Additionally the evolution with time of the pore water pressure in the corner where failure initiates is plotted in Fig.7. The suction at the initiation of failure is represented by the dots and is comprised between 225 and $400 \mathrm{kPa}$ depending on the test. Such values are very close to the air-entry suction of the material (around $400 \mathrm{kPa}$ according to the retention data shown in Fig.3), which is consistent with other experimental evidence showing that cracking occurs at the transition from saturated to unsaturated states $[17,18]$.

Table 2. Comparison between experimental and numerical time of cracking.

\begin{tabular}{l|l|l}
\hline & Experiment & Simulation \\
\hline Test 1 & $3 \mathrm{~h} 28^{\prime}$ & $3 \mathrm{~h} 26^{\prime}$ \\
\hline Test 2 & $4 \mathrm{~h} 37^{\prime}$ & $3 \mathrm{~h} 30^{\prime}$ \\
\hline Test 3 & $5 \mathrm{~h}$ & $5 \mathrm{~h} 32^{\prime}$ \\
\hline Test 4 & $2 \mathrm{~h} 18^{\prime}$ & $2 \mathrm{~h} 22^{\prime}$ \\
\hline
\end{tabular}

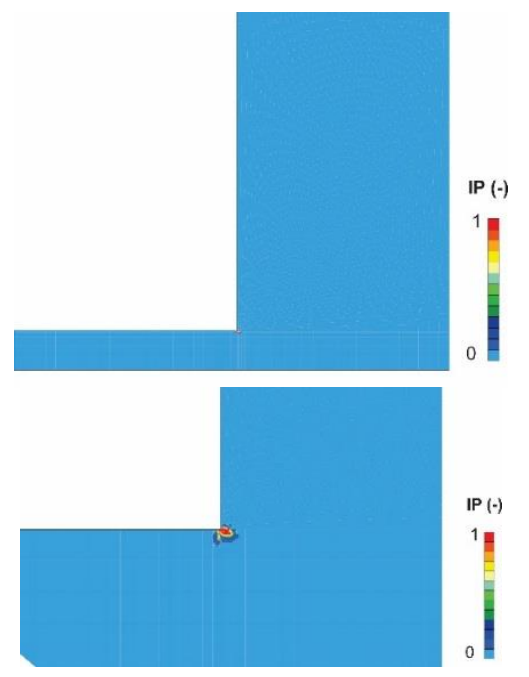

Fig. 5. Plastic index IP on top surface for test 3 after $6 \mathrm{~h}(\mathrm{IP}=1$ means that the shear failure criterions is reached) (a) for the whole sample and b) zoom around the corner where the onset of cracking is observed. 


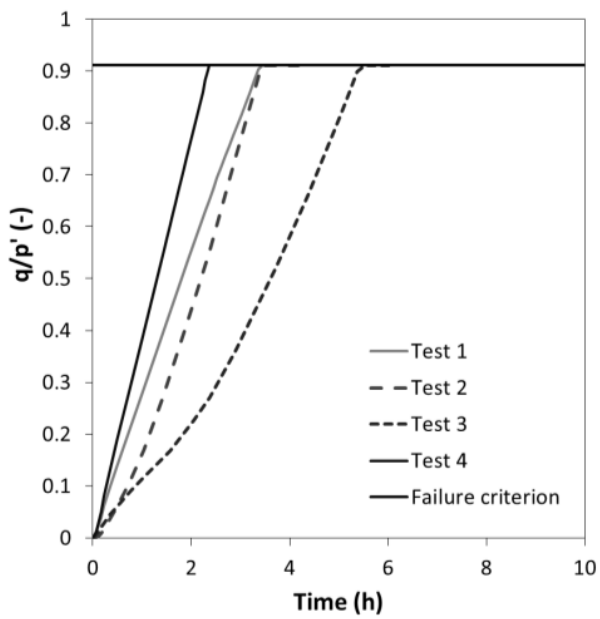

Fig. 6. Evolution with time of the ratio between deviatioric stress $q$ and effective mean stress $p$ ' at the corner between 'web' and the 'flange' (on the top surface) for the 4 bars.

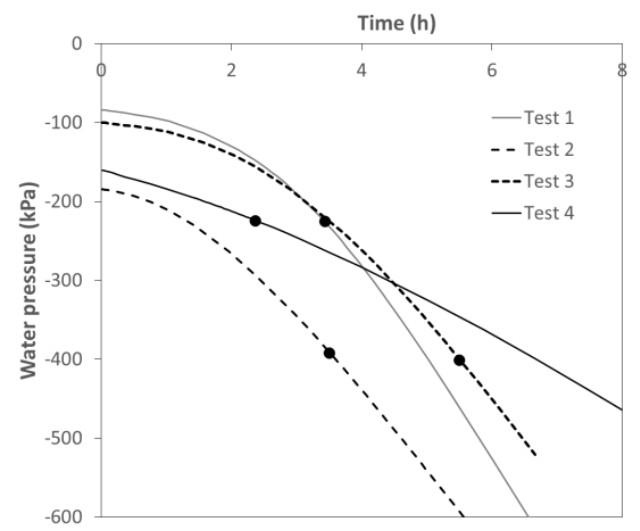

Fig. 7. Water pressure evolution at the corner between the 'web' and the 'flange' (at the top surface). Dots represent the numerical onset of cracking.

\section{Conclusions}

This paper has investigated the mechanisms of tensile failure in clayey materials under total tensile stresses, but effective compressive stress states. To this end, finegrained soil bars with complex geometries and nonuniform boundary conditions (constrained shrinkage, evaporation prevented on some surfaces) were first subjected to air-drying. The time and location of cracking observed experimentally were then compared with time and location of cracking predicted by a 3-D hydromechanical simulation using a relatively simple nonelastic constitutive law and classical fluid transfer equations for unsaturated porous media. The onset of cracking was assumed to occur when the effective stress state reaches a cohesionless shear failure criterion suitable also to interpret direct tensile tests in terms of effective stresses. All the soil parameters of the model have been preliminary determined from independent geotechnical tests, so that the results of the simulations can be considered as "blind predictions".
The hydro-mechanical simulations suggest that nonlinear elasticity associated with shear failure criterion is able to predict both the time and the location of cracking. This allowed validating the assumption that cracking can be explained by an effectives-stress dependent failure criterion, i.e. assuming that crack initiates by shearing (Mode II) under tensile total stress states. This finding supports therefore recent conclusions in the literature $[8$, 9] around this issue of the failure mechanisms under total tensile stress.

Nevertheless, additional simulations should be performed to investigate some remaining open questions, as the effect of small imperfections in the geometry of the bar on the cracking. New geometries of the bars with other non-uniform mechanical restraints and evaporation conditions that can generate cracking in other locations that the corner can be also relevant to fully validate the ability of the model to predict the location of cracking.

\section{References}

1. R. Baker, Soils Found. 21, 7 (1981)

2. E. Marnette, C. Schuren, K. van de Brink, N.D. Glew, M. Dyer, Advanced experimental unsaturated soil mechanics : Experus 2005 (2006)

3. J.M. Konrad, R. Ayad, Can. Geotech. J. 34 (1997)

4. C.S. Tang, B. Shi, C. Liu, L. Gao, H.I. Inyang, J. Mater. Civil Eng. 23, 6 (2011)

5. A. Corte, A. Higashi, Research Report 66: U.S. Army Snow Ice and Permafrost Research Establishment. (1960)

6. H. Péron, T. Hueckel, L. Laloui, L.B. Hu, Can. Geotech. J. 46 (2009)

7. M. Sanchez, O.L. Manzoli, L.J.N. Guimarães, Comput. Geotech. 62 (2014)

8. N.I. Thusyanthan, W.A. Take., S.P.G. Madabhushi, M.D. Bolton, Géotechnique. 57, 7 (2007).

9. I. Murray, A. Tarantino, Géotechnique, in press. (2018)

10. F. Collin, X.L. Li, J.-P. Radu, R. Charlier, Engng. Geol. 64 (2002)

11. P. Gerard, R. Charlier, R. Chambon, F. Collin. Water Resour. Res. 44, 5 (2008).

12. P. Gerard, R. Mpawenayo, M. Douzane, F. Debaste, Environ. Geotechnics, in press (2018)

13. R. Charlier, F. Collin, B. Pardoen, J. Talandier, J.-P. Radu, P. Gerard, Engng. Geol. 65 (2013)

14. M.T. van Genuchten, Soil Sci. Soc. Am. J. 44 (1980)

15. M. Nuth, L. Laloui, Int. J. Numer. Anal. Met. 32 (2008)

16. E.E. Alonso, J.M. Pereira, J. Vaunat, S. Olivella, Géotechnique. 60, 12 (2010)

17. G.W. Scherer, Theory of drying. J. Am. Ceram. Soc. 73, 1 (1990)

18. P.G. Simpkins, D.W. Johnson, D.A. Fleming, $J$. Am. Ceram. Soc. 72, 10 (1989). 